\title{
Diagnosis of pneumonia by community health volunteers: Experience of BRAC, Bangladesh
}

\author{
Abdullahel Hadi $M S D P H$ \\ Research and Evaluation Division \\ BRAC, 75 Mohakhali, Dhaka 1212, Bangladesh
}

Correspondence

Abdullahel Hadi

Research and Evaluation Division

BRAC

75 Mohakhali

Dhaka 1212, Bangladesh

Fax: 880-2-8823542

e-mail: shirsha@bangla.net 


\title{
Diagnosis of pneumonia by community health volunteers: Experience of BRAC, Bangladesh
}

\begin{abstract}
SUMMARY
The study assessed the competency of community health volunteers in diagnosing pneumonia in children through simple clinical signs. Data were collected by a group of research physicians who observed the case management performance of 120 health volunteers in Bangladesh where BRAC has been providing community-based acute respiratory infection (ARI) control programme since mid 1992. About 1,166 children aged 3-60 months were diagnosed at household level using simple signs. Findings revealed that the health volunteers' competency in correctly diagnosing pneumonia and no pneumonia was quite high although they had difficulties in diagnosing severe and very severe pneumonia cases. Among the clinical signs such as coarse breath sounds and chest in-drawing, the correct diagnoses of pneumonia was lower than no pneumonia. Overall agreement rates for most signs were very high. The study concludes that the less educated health volunteers can also be effectively used in diagnosing pneumonia at grassroots level in developing countries.
\end{abstract}




\section{INTRODUCTION}

Acute respiratory infections (ARIs) are the leading causes of morbidity and mortality in infancy and childhood in most developing countries. ${ }^{1.5}$ Among them, pneumonia alone accounted for nearly $75 \%$ of all ARI deaths. ${ }^{4}$ The WHO initiated ARI control programme in mid $1980 \mathrm{~s}$ focussing on the identification and treatment of pneumonia cases by community health volunteers. ${ }^{4,6}$ But not many countries were able to launch and implement such a programme. Lack of accurate and timely diagnosis of pneumonia has remained as a major problem in primary health care systems particularly in poor countries.

Nearly 400 children die each day from ARIs in Bangladesh. ${ }^{7}$ BRAC, a non-government development organisation in Bangladesh, has been trying to develop a simple and sustainable model to control and treat acute respiratory infection (ARI) through its community health volunteers. $^{8}$ The part-time community health volunteers had little formal schooling. They, however, received both pre and in-service training in diagnosing and treating children with ARI and were working for more than five years. In identifying the potential cases in their assigned villages, simple clinical signs of pneumonia in children were used by the volunteers as also found in many studies. ${ }^{4.59}$ The purpose of the present study to assess the competency of community health volunteers in diagnosing pneumonia at the grassroots level and examine the effectiveness of clinical signs used by BRAC volunteers in diagnosing pneumonia in rural Bangladesh.

\section{METHODS AND MATERIALS}

The study was conducted in villages where BRAC has been providing community-based ARI control programme since mid 1992. The programme covered 2.4 million population in 10 subdistricts in northern and central regions of Bangladesh. The study was designed to be representative of all health volunteers where BRAC provided ARI control programme. Thus, 12 volunteers from each of the 10 sub-districts were selected following systematic random sampling technique to yield a total of 120 volunteers. The diagnosis of the volunteers was observed at the households by the research physicians of BRAC. Five research physicians were trained in ARI case management in a specialised child hospital. A team of one health volunteers and one research physician was sent to the selected community to identify and diagnose the potential cases. In total, the research teams examined 1,166 children with cough and common cold. 
In the sample villages, each health volunteer visited her assigned households with the research physician. Not all children were eligible to be examined. After verbal consent of the child's caretaker, only children aged 3 months to 5 years with cough, common cold and runny nose were considered to be included in the sample. Children were examined first by the community health volunteer and then by the research physician. The research physicians observed and document the clinical diagnosis of each health volunteer. Then the research physician himself examined each child following the WHO guidelines. The examinations included measurement of respiratory count, assessment of chest in-drawing, difficult breathing, body temperature, shrunken eyes and the history of convulsion. The reports were then compared to evaluate the performance of the health volunteers. Data were collected during December 1998 through February 1999. One drawback of this approach was that health volunteers might have tried to perform better than their normal performance in the presence of a research physician. The effect, however, was expected to be minor since physicians had to develop friendly relationship with the health volunteers.

Indicators such as health volunteers' correct diagnosis of pneumonia, correct diagnosis of no pneumonia, positive predictive values and overall agreement rates were calculated through $2 \times 2$ contingency tables. McNemar tests were considered appropriate to assess the differences in diagnosis.

\section{RESULTS}

Both the community health volunteers and research physicians examined 1,166 children. The mean age of the study children was nearly 20 months (ranging 3-60 months). Of them, 56\% were boys. Significant differences in diagnosis were observed between the health volunteers and physicians (Table 1). The health volunteers identified about $19 \%$ children to have pneumonia of any kind while the physicians found $22.6 \%$ pneumonia cases. The differences in each pneumonia classification indicated a tendency among the health volunteers to under-report potential cases. The differences in diagnosis, however, were not statistically significant in very severe and severe cases probably because of small sample sizes.

Table 1 here

\section{7}


Table 2 shows that the validity of health volunteers' diagnosis in identifying pneumonia by the health volunteers were reasonably high compared to findings in other studies. ${ }^{1.5}$ When the rates were differentiated by classification, it appeared that only $12.5 \%$ of the very severe pneumonia cases were correctly diagnosed. The sensitivity or the correct diagnosis of pneumonia, however, increased to $45.8 \%$ in severe and about $65.8 \%$ in mild pneumonia cases. The specificity or the correct diagnosis of no pneumonia remained very high. The positive predictive value or the proportion of health volunteers' diagnosis of pneumonia that were truly pneumonia was $80.5 \%$ Like sensitivity, the predictive values varied widely by classification of pneumonia.

Table 2 here

Not all clinical signs were sensitive in identifying ARI cases. In Table 3, the clinical signs such as the respiratory rate, breathing difficulty and chest in-drawing were found good indicators to identify pneumonia. A child was considered to have respiratory problem if i) more than 50 breaths/min were counted among children aged 2-11 months; and ii) more than 40 breaths/min were counted for children between 12 and 60 months. Signs like convulsion and shrunken eyes were not found as good indicators to be used by the health volunteers as none of them was able to correctly identify any case. Unlike other studies, ${ }^{5}$ the sensitivity and the predictive value of body temperature were found very high in this study. The specificity rates for the clinical signs were also very high except the body temperature.

The findings clearly demonstrate that the health volunteers emphasised more on 'not to provide wrong treatment' than 'providing no treatment' to the children. Overall agreement rates among the health volunteers and physicians were very high (>90\%) for most of the signs indicating that the aggregate variability in correct diagnosis of pneumonia and no pneumonia between the health volunteers and physicians was minimum.

Table 3 here

\section{DISCUSSION}

The pneumonia control and treatment at the grassroots level has rarely been tried probably because the routine screening of ARI cases by paramedics or health professionals was considered too expensive in most developing countries. This study has questioned this assumption and 
demonstrated that less educated women volunteers can also be effectively used in identifying potential pneumonia cases. Results show, however, that the health volunteers had difficulties in identifying severe and very severe cases and that a proportion of the children might not received appropriate treatment as a result. Nevertheless, correct diagnoses of pneumonia and no pneumonia at the aggregate level suggested that the approach adopted by BRAC worked well that could encourage others to design a low-cost ARI control programme in poor communities.

No single clinical sign was considered decisive to diagnose pneumonia although some clinical signs were found more powerful than others. ${ }^{1,49-10}$ The study focused on multiple signs rather than single ones and attempted to identify the more sensitive signs in the community settings. As found in other studies, ${ }^{3-5}$ respiratory rate, difficult breathing and body temperature correlated well with the diagnosis of the physicians. While chest in-drawing was also proved to be an important sign of pneumonia, health volunteers had difficulty to effectively use it. Results also validated earlier works that respiratory rate and difficult breathing were reliable signs for the diagnosis of pneumonia. Among other clinical features, sign like convulsion or shrunken eyes were poorly diagnosed by the community volunteers in Bangladesh. The results also confirmed other works that rapid breathing in children is a useful sign for identifying pneumonia. ${ }^{3}$

The study concludes that the simple clinical signs-based case assessment algorithm for pneumonia, as practised in BRAC, can be very effective in diagnosing pneumonia in children. The primary heath care programmes in countries with substantial child mortality could benefit from this experience in Bangladesh.

\section{ACKNOWLEDGEMENTS}

This research was a part of the evaluation of ARI Control Project of BRAC, Bangladesh. The help of Dr. Gail Davey of the St. George Hospital Medical School of the UK in analysing the data is gratefully acknowledged. The author wishes to thank the community health volunteers and the research physicians who had difficult times in collecting information for this research. 


\section{REFERENCES}

1. Harrison LH, Moursi S, Guinena AH, Gadomski AM, El-Ansary KS, Khallaf N, Black RE. Maternal reporting of acute respiratory infection in Egypt. Int J Epidemiology 1995; 24:1058-63.

2. Zaman K, Baqui AH, Yunus M, Sack RB, Bateman OM, Chowdhury HR, Black RE. Acute respiratory infections in children: a community-based longitudinal study in rural Bangladesh. J Trop Pediatrics 1997; 43:133-37.

3. Redd SC, Vreuls R, Metsing M, Mohobane PH, Patrick E, Moteetee M. Clinical signs of pneumonia in children attending a hospital outpatient department in Lesotho. Bull WHO 1994; 72:113-18.

4. Gupta D, Mishra S, Chaturvedi P. Fast breathing in the diagnosis of pneumonia - a reassessment. $J$ Trop Pediatrics 1996; 42:196-99.

5. Harari M, Shann F, Spooner V, Meisner S, Carney M, Campo JD. Clinical signs of pneumonia in children. Lancet 1991; 338:928-30.

6. World Health Organization. A programme for controlling acute respiratory infections in children: memorandum from a WHO meeting. Bull WHO 1984

7. Government of Bangladesh. Report on ARI Health Facility Survey. Dhaka: Government of Bangladesh. National ARI control programme.1995.

8. BRAC. Annual Report 1997. Reproductive Health and Disease Control Program. 1998.

9. Simoes EA, McGrath EJ. Recognition of pneumonia by primary health care workers in Swaziland with a simple clinical algorithm. Lancet 1992; 340:1502-3.

10. Pereira JCR, Escuder MML. The importance of clinical symptoms and signs in the diagnosis of community-acquired pneumonia. $J$ Trop Pediatrics 1998; 44:18-24. 
Table 1. Proportion of pneumonia cases by classification

\begin{tabular}{llll}
\hline \multirow{2}{*}{$\begin{array}{l}\text { Pneumonia } \\
\text { classification }\end{array}$} & \multicolumn{2}{c}{ Diagnosed by } & \\
\cline { 2 - 3 } & Health volunteer & Physician & P-value* \\
\hline All & & & 0.000 \\
Any pneumonia & $18.9(221)$ & $22.6(263)$ & \\
No pneumonia & $81.1(945)$ & $77.4(903)$ & \\
& & & \\
Classification & & & \\
$\quad$ Very severe & $0.3(4)$ & $0.7(8)$ & 0.344 \\
$\quad$ Severe & $1.7(20)$ & $2.1(24)$ & 0.523 \\
$\quad$ Mild & $16.9(197)$ & $19.8(231)$ & 0.003 \\
& & & \\
$\mathrm{~N}$ & 1,166 & 1,166 & \\
\hline
\end{tabular}

Frequencies are shown in the parentheses

* P-values are estimated by using McNemar test. 
Table 2. Diagnosis of pneumonia cases by classification among children

\begin{tabular}{lll}
\hline $\begin{array}{l}\text { Validity of } \\
\text { health volunteers' } \\
\text { diagnosis }\end{array}$ & $\begin{array}{l}\text { Research physician diagnosis } \\
\text { Pneumonia } \quad \text { No pneumonia }\end{array}$ & $\begin{array}{l}\text { Positive } \\
\text { predictive } \\
\text { value }\end{array}$ \\
\hline
\end{tabular}

All

Any pneumonia

$67.7(178) \quad 4.8 \quad(43)$

80.5

No pneumonia

$32.3(85)$

$95.2(860)$

Classification

Very severe

Pneumonia

No pneumonia

12.5 (1)

87.5 (7)

$0.3 \quad$ (3)

25.0

Severe

Pneumonia

45.8 (11)

No pneumonia

54.2 (13)

0.8 (9)

55.0

Mild

Pneumonia
No pneumonia

65.8 (152)

99.2 (1133)

34.2 (79)

4.8 (45)

$95.2(890)$

Frequencies are shown in the parentheses 
Table 3. Diagnosis of clinical signs of pneumonia among children

\begin{tabular}{|c|c|c|c|c|}
\hline \multirow{2}{*}{$\begin{array}{l}\text { Validity of individual } \\
\text { signs of health } \\
\text { volunteers' diagnosis }\end{array}$} & \multicolumn{2}{|c|}{ Research physician diagnosis } & \multirow{2}{*}{$\begin{array}{l}\text { Positive } \\
\text { predictive } \\
\text { value }\end{array}$} & \multirow{2}{*}{$\begin{array}{l}\quad \% \\
\text { Overall } \\
\text { agreement }\end{array}$} \\
\hline & Pneumonia & No pneumonia & & \\
\hline Respiratory rate & $58.7(37)$ & $8.9(36)$ & 50.7 & 86.7 \\
\hline No respiratory rate & $41.3(26)$ & $91.1(369)$ & & \\
\hline Noisy breathing & $59.1(13)$ & $3.3(12)$ & 52.0 & 94.5 \\
\hline No noisy breathing & $40.9(1)$ & $96.7(347)$ & & \\
\hline Chest in-drawing & $60.0(3)$ & 2.0 & 38.5 & 98.1 \\
\hline No chest in-drawing & $40.0(2)$ & $98.0(400)$ & & \\
\hline Convulsion & $\cdots \quad(0)$ & $1.6(3)$ & -- & 98.0 \\
\hline No convulsion & $\cdots \quad(3)$ & $98.4(179)$ & & \\
\hline Shrunken eye & $-\quad(0)$ & 1.1 (3) & -- & 98.5 \\
\hline No shrunken eye & $-\quad(1)$ & $98.9(261)$ & & \\
\hline Body temperature & $89.7(583)$ & $18.7(20)$ & 96.5 & 88.4 \\
\hline No body temperature & $10.3(68)$ & $81.3(87)$ & & \\
\hline
\end{tabular}

Frequencies are shown in the parentheses

-- Number of cases is too small. 